\title{
Analysis of surface electromyography for hand gesure classification
}

\author{
Najla Ilyana A.M, Nor Aini Z, Sharvin R, Norlaili M.S \\ Biomedical Instrumentation and Electronics research Group, School of Electrical Engineering, Faculty of Engineering, \\ Universiti Teknologi Malaysia, Malaysia
}

\begin{tabular}{|c|c|}
\hline Article Info & ABSTRACT \\
\hline Article history: & \multirow{9}{*}{$\begin{array}{l}\text { Electromyography (EMG) is the measure of electrical activity produced by } \\
\text { skeletal muscle. It is useful in prosthetic and rehabilitation technology as } \\
\text { well as ability to handle electronic devices and robotics. If the EMG signal } \\
\text { from the body especially hand movement can be apprehended, better value } \\
\text { for people all around the world can be provided. Furthermore, it can be used } \\
\text { to control smart-phone and be integrated with wearable technology. Another } \\
\text { interesting application of this technology is in sign language recognition } \\
\text { which is able to assist many disabled people in their daily lives. In this paper, } \\
\text { hand gesture signals are acquired, extracted, analysed and classified. The } \\
\text { EMG data from hand gesture which are rock, paper and scissors managed to } \\
\text { be extracted. We use time domain feature to classified using Principal } \\
\text { Component Analysis and regression tree. The result was highly accurate with } \\
72.59 \% \text { and } 80.85 \% \text { for PCA and regression tree respectively. }\end{array}$} \\
\hline Received Dec 28, 2018 & \\
\hline Revised Mar 30, 2019 & \\
\hline Accepted Apr 25, 2019 & \\
\hline Keywords: & \\
\hline Hand gesture & \\
\hline Principal component analysis & \\
\hline Regression tree & \\
\hline Sign language recognition & \\
\hline Wearable technology & \\
\hline
\end{tabular}

\section{Corresponding Author:}

Najla Ilyana binti Ab Majid,

Biomedical Instrumentation and Electronics Research Group,

School of Electrical Engineering,

Faculty of Engineering, Universiti Teknologi Malaysia (UTM),

Skudai, 81310, Johor, Malaysia.

Email: nilyana8@live.utm.my

\section{INTRODUCTION}

Human computer interaction has become a vital aspect in human life due to advancement of technology in augmented reality, virtual reality and wearable technology. Hence, the need of technology that can integrate with our own body and gesture has become prominent. Nowadays, it is possible to integrate full computer in the size of our hand as well as creating technologies which can process at high speed with low cost and low power consumption. This allow us to bring intelligence close to our fingertips and make it able to carry everywhere. Thus, it is possible to be able to create a wearable or portable device with enough processing power as well as able to compute and interpret data from electrodes or sensors to deliver fast and accurate data.

There are a lot of technologies can interpret hand gestures or movement. A wearable technology based on vision to recognize hand movement was proposed in [1-2]. It is a combination of an integrated Analog Front End and embedded processing which provides high quality signal acquisition and on-board digital processing capabilities with a contained power consumption. However, it requires camera to be mounted in the environment which inherently suffer from a limited range of vision as well as expensive to implement and privacy issue. Wu et al (2016) has proposed a glove-based SLR system to recognize sign language by the combination of IMU and sEMG sensors. The device is accurate and able to detect the slightest change in movement [3-5]. However, the device is costly and requires a lot of sensors which is uncomfortable and unpractical for daily life usage. 
Recently, IMU technology is gaining popularity due to its low cost and less power consumption. A study in [6-8] proposed a wearable technology to detect gesture based on IMU system. However, IMU sensor has limitation to detect the fine motion of a hand and fingers. It has limitation to detect more hand gestures due to its measurement only for acceleration and gravity from 3-axis. Surface electromyography (sEMG) is a tool to collect biological electrical signal during neuromuscular activity from the skin surface through electrode. The signal was then amplified, displayed and recorded. It exists in different degree of correlation with muscle function and activity states that reflect neuromuscular activity at some level. sEMG is a noninvasive, real time and multiple target, neuromuscular disease diagnosis in clinical medicine, rehabilitation medicine in the field of muscle function evaluation and fatigue assessment in sports science, exercise technology and analysis [9]. There are many researches focuses on pattern recognition of sEMG to differentiate limb motion.

The EMG signals were recorded and classified using K-Nearest Neighbours (K-NN) and Linear Discriminant Analysis (LDA) where LDA has become the most accurate classifier method obtained [10-12]. A portable sEMG sensor however can measure the hand gesture through the acquisition of muscle electrical activity using less sensor requirement [2], [13-16].

However, the complexity of electromyography signal produce causes the arm motion state difficult to determine. Studies on biological signals have provided interpretation of EMG signals and new methods for modelling and classifying hand movements. A control system must have accurate, intuitive control and acceptable response time to be successful. Hence, it is important for the control system with almost $100 \%$ accuracy so that user can perform the desired movement. The response time of the control system should be short enough for the user to understand [3].

In this study, we intend to create a system that can recognize basic hand gestures using two channel surface EMG signal where the system used different classification techniques which are Principle Component Analysis (PCA) and classification and regression tree using distinctive time domain features. The sEMG electrodes are located at the forearm to acquire signal which will then need to be extracted, analyzed and classified to interpret the detection of hand gestures which are rock, paper and scissors.

The system proposed is a low cost and require simple analysis of hand gesture. Many publications used time domain as the main tool to extract features due to its obvious advantage from frequency domain [17]. However, each author used different time domain feature to recognize the body movement. PCA is a less computational requirement which uses Euclidean distance equation and the classification and regression tree was used to measure the purity of the results [18-20]. The study used time domain as a feature extraction tool due to its nature of having good discrimination power and less computationally intensive.

The rest of the paper is organized as follows: In part II, a methodology of the research is presented including the experimental setup; Part III is a presentation of results and discussion; Part IV gives a conclusion.

\section{RESEARCH METHOD}

Figure 1 shows the block diagram for hand gesture classification system. Five healthy samples that do not have damage in their right arm participated in the project. The subjects are chosen from the age range 20 to 25 years old. The subject was asked to sit and relax while placing the arm on the lap. The arm was wiped with alcohol swab to remove dirt and sweat. The electrodes were then placed with some EMG paste to increase conductivity of the electrode. The measuring signal was start after 30 to 60 seconds. The electrodes are placed at the forearm which is near to flexor carpi radialis longus muscle as shown in Figure 2. It is based on most researchers due to ability to get high recognition rate from this muscle [3], [21-23]. The bipolar configuration was used to improve the signal to noise ratio $(\mathrm{SnR})$ of the sEMG signal reading. The electrodes were placed $2 \mathrm{~cm}$ apart from each other.

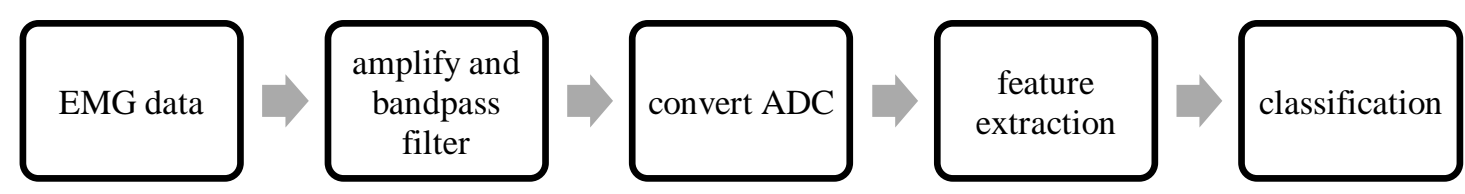

Figure 1. Block diagram for hand gesture classification system 

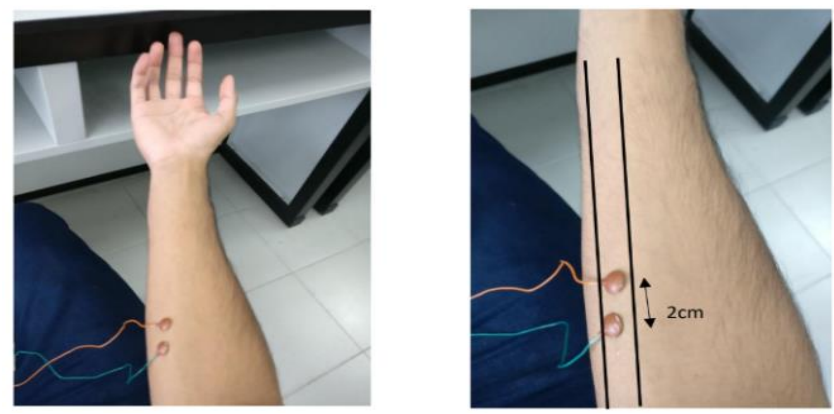

Figure 2. Electrode placement with bipolar configuration

The signal acquired were feed to BMA-400 AC/DC low noise basic amplifier and transducer to amplify the biomedical signals from the electrodes. It has extremely low noise output, sharp cut-off bandpass filters and wide gain range as well as having high impedance instrumentation differential amplifiers to filter and isolate initial noise. The signal was then converted into digital using Analog to Digital Converter, USB6009. It is low cost multi-function DAQ device from National Instrument to convert analogue signal from transducer and amplifier to be converted into digital.

Each subject was asked to perform hand gesture as in Figure 3 which are rock, paper and scissors. They need to repeat the hand gestures for 25 times each. The experiment was repeated three times corresponding each gesture. The data collection was observed to ensure there will be unobvious errors made during the process. Each gesture was repeated at a time interval of 4 seconds which resulted 60 to 70 seconds total recording time for each gesture. The sampling frequency used was $1000 \mathrm{~Hz}$. at the end of experiment, the data from three samples, while the rest of the data were corrupted by noise and unusable.

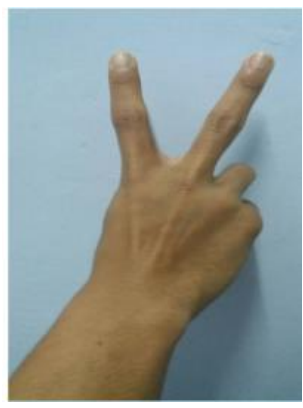

(a)

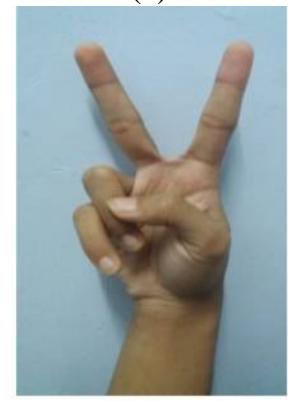

(d)

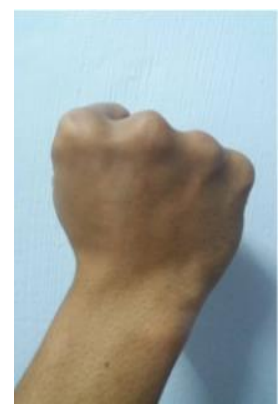

(b)

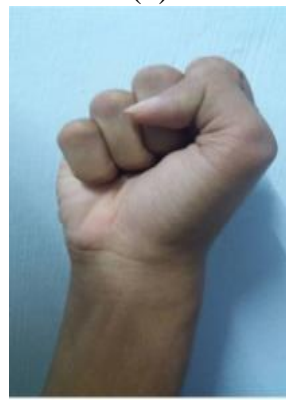

(e)

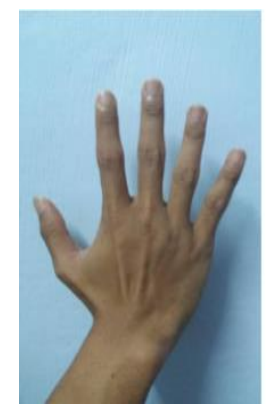

(c)

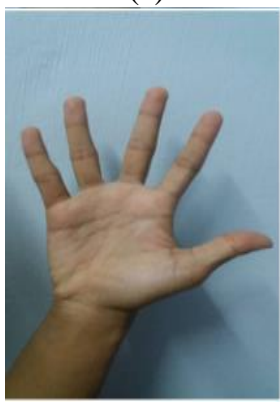

(f)

Figure 3. Hand gestures a) scissor back hand b) rock back hand c) paper back hand d) scissor front hand e) rock front hand f) paper front hand

Raw EMG data always suffer from noise should be removed to ensure good classification rate. The raw data was sent to MATLAB to be filtered, rectified and any DC offset were removed. A 2nd order Butterworth band-pass filter with $10-500 \mathrm{~Hz}$ cut off frequency was used. Figure 4 shows the signal before and after filtration process. DC offset was removed using 'detrend' function in MATLAB. 


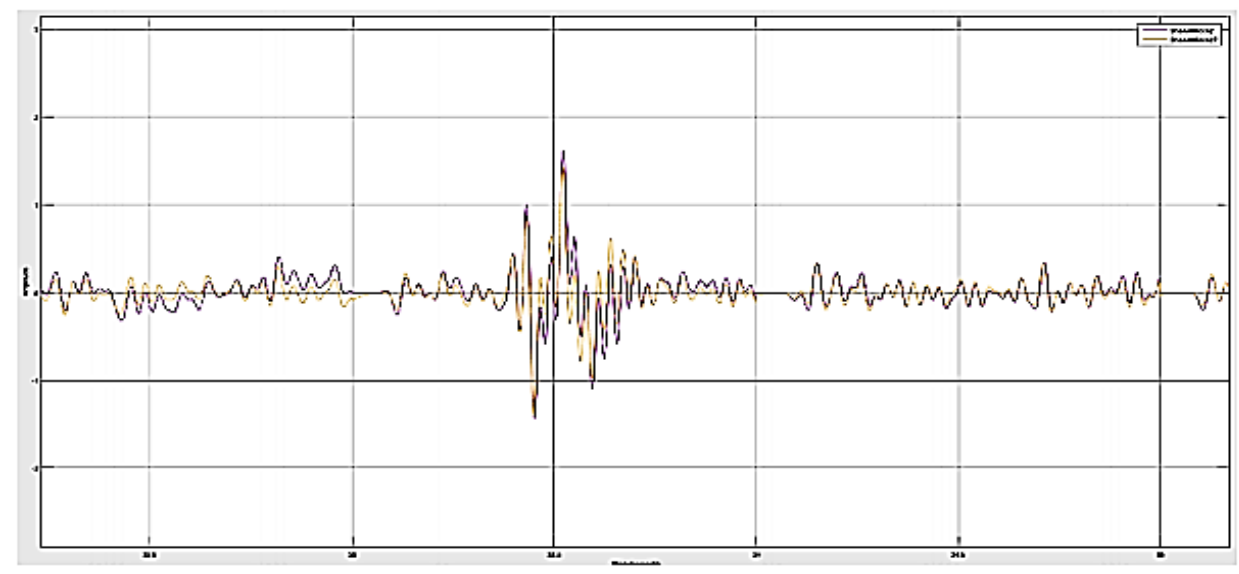

Figure 4. Signal before and after filter

The most crucial step in classification of EMG signal is the feature extraction process as the accuracy and realty of classification process relies heavily on the selection of features. In this study, we used time domain instead of frequency domain due to the most preferable by most papers which is having a good discrimination power and less computationally intensive. There are large number of features have been proposed and studied for both sEMG and IMU sensors for detecting activities and gestures. Hence, we adopted some of well-established features [3], [15], [21-25]. A set of statistical features was obtained from EMG signal which are mean, mean absolute value, standard deviation, variance, skewness, number of zero crosses and root mean square.

After the extraction process, a good recognition system is required to classify the data by using a good classifier translates to a better classification rate. Classification is a process of sorting objects into predetermined group which is the combination of the nearest alternatives to ease the data evaluation and characterize them. In this study, we used Principal Component Analysis (PCA) and Regression Tree.

Principal Component Analysis (PCA) is a dimensionally reduction technique that is mostly used to change a high dimensional dataset into smaller dataset after using machine learning algorithm to study the data. The classifier requires enormous computation for matrix operation but using the Simple-PCA which can calculate eigenvectors has been proposed to reduce the computational complexity without using matrix operation [9]. It is a multivariable classifier which helps to reduce the size of the data set and have fewer dimension to be analyzed. Regression tree predicts continuous variables or regression and characterized them based on nodes. It is one of the oldest and powerful classification techniques which basically works by splitting up choices in a tree format that helps with predictive analysis.

\section{RESULTS AND ANALYSIS}

In this study, we consider seven time domain features which were extracted from the data as follows: (1) Mean- average value of the data, (2) Mean absolute value- to measure the gauge muscle contraction strength, (3) Standard deviation- measure variability from the mean of signal, (4) Variancemeasure of how far each value in the data set is from the mean, (5) Skewness- measure the symmetry of the data assuming a non-Gaussian distribution in the data, (6) Number of zero crosses- measure amount of time the signal value changes sign and (7) Root mean square- measure of the signal energy normalized by the number of samples.

The results show that both PCA and regression tree results were accurate in detecting three hand gestures which are paper, rock and scissors with $72.59 \%$ and $80.85 \%$ respectively.

\subsection{PCA}

The eigenvalues of PCA was managed to be found and displayed in the Figure 5. Figure 5 a) shows the scree plot of the fraction of total variance in the data which shows the cumulative percentage of each fraction, while Figure $5 \mathrm{~b}$ ) the contribution of variables for F1 and F2. F1 and F2 were derived from the features extracted from the raw EMG data and tabulated which is shown in a low dimensional map for optimal view. Based on the Figure 5 b) most of the variables contributed by F1 which was then tabulated in Figure 6 that shows the value of variable contributed by F1. 
The dimensional map of F1 and F2 shows that most of the features lies on the positive $\mathrm{x}$-axis which is F1. Its shows that F1 contribute most features. Figure 6 shows that the strongest features contributed for F1 are RMS, Variance, Standard Deviation and MAV with 24.4\%, 23.9\%, 24.4\% and 20.9\% respectively. The NZR and Skewness contribute $3.1 \%$ each while mean only contribute $0.2 \%$ to F1.
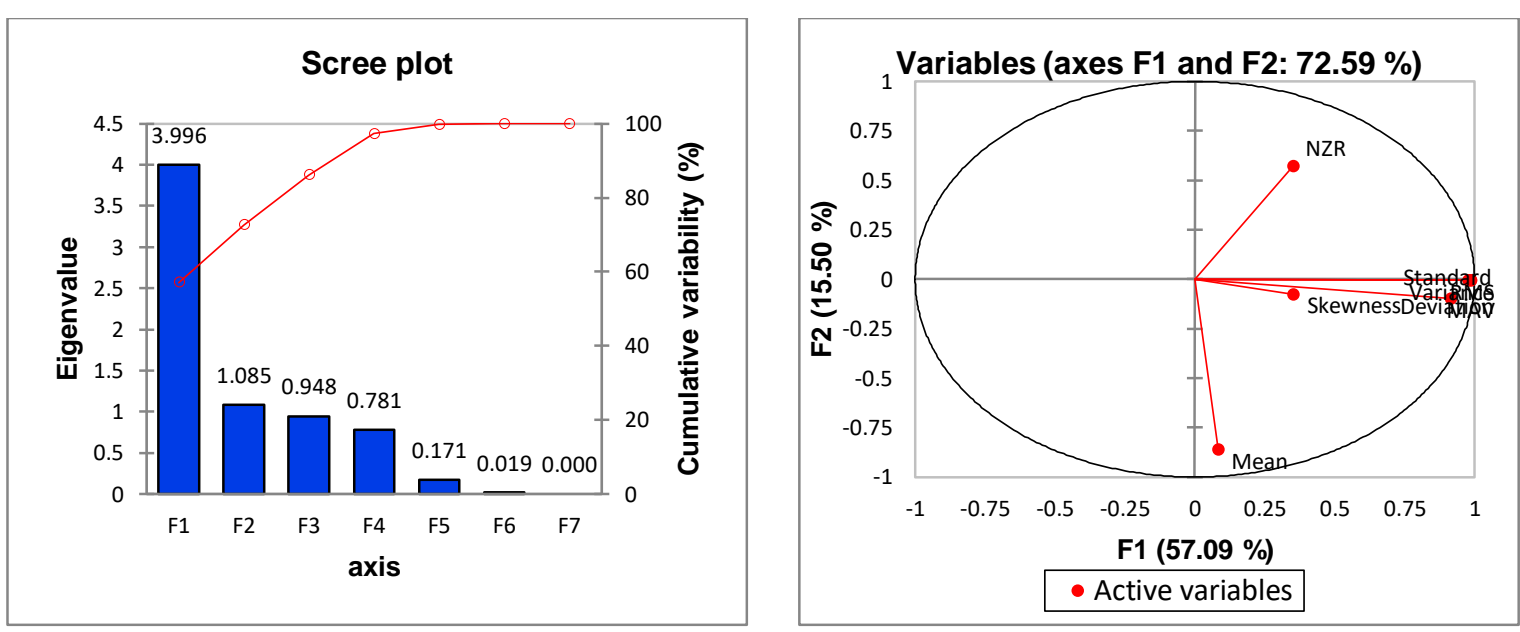

Figure 5. a) Scree Plot b) Dimensional map for F1 and F2

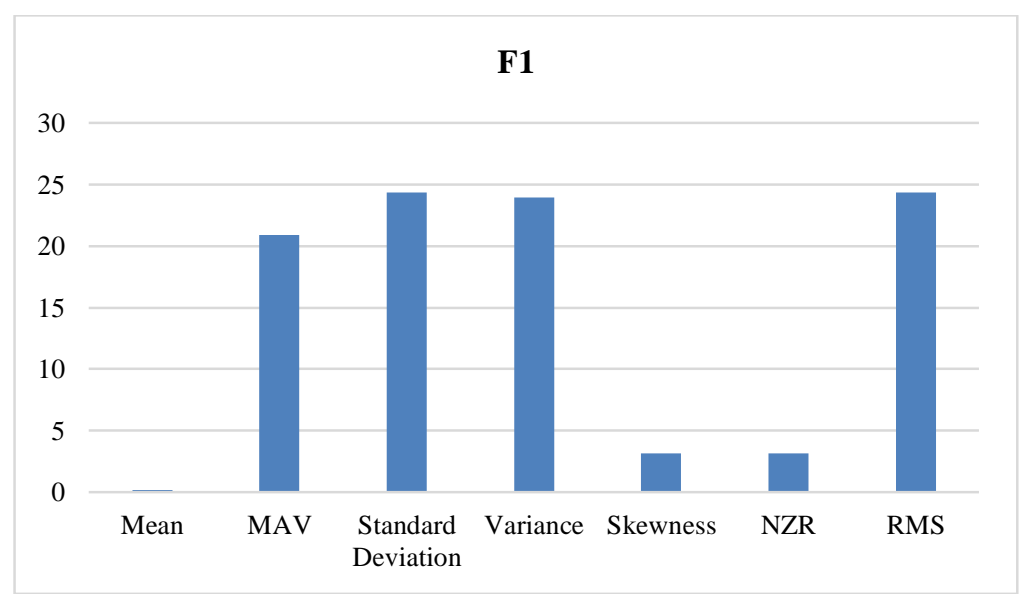

Figure 6. Variable distribution for F1

\subsection{Regression Tree}

Table 1 shows the confusion matrix for the training sample where the classification and regression tree help the purity process of the raw data based on its predictive analysis and does a new predictive model to observe the data accuracy. Figure 7 shows a decision tree map of possible outcomes of the signal choices. The tree map was initially divided into nine nodes by variable MAV.

From the Table 1, the highest accuracy obtained by the classifier is rock with $92.59 \%$. The lowest accuracy is scissors with $72.50 \%$ where most of them were detected as paper. This was mainly due to inability to differentiate scissors and paper. The movement of scissors and rock are almost the same which using only two fingers.

\subsection{Future Work}

Based on the PCA results, there are only four strongest features can be used for future work to classify the signals. sEMG is limited to only record down the biological electrical signal of neuromuscular activity with different degree of great randomness and uncertainty, we intend to further this study to add another wearable sensor to detect hand movement from three axis attach on upper limb. Other features and 
classifiers also can be added in the future work to verify the additional sensor as well as recruiting more subjects in the experiment.

Besides, more data samples should be included for better prediction model. Since the entire system was managed to build from scratch and was usable, so we can focus more on the data collection part and more raw data can be obtained. Noise plays very important role in this experiment; thus, better noise reduction technique can be used to enhance the results.

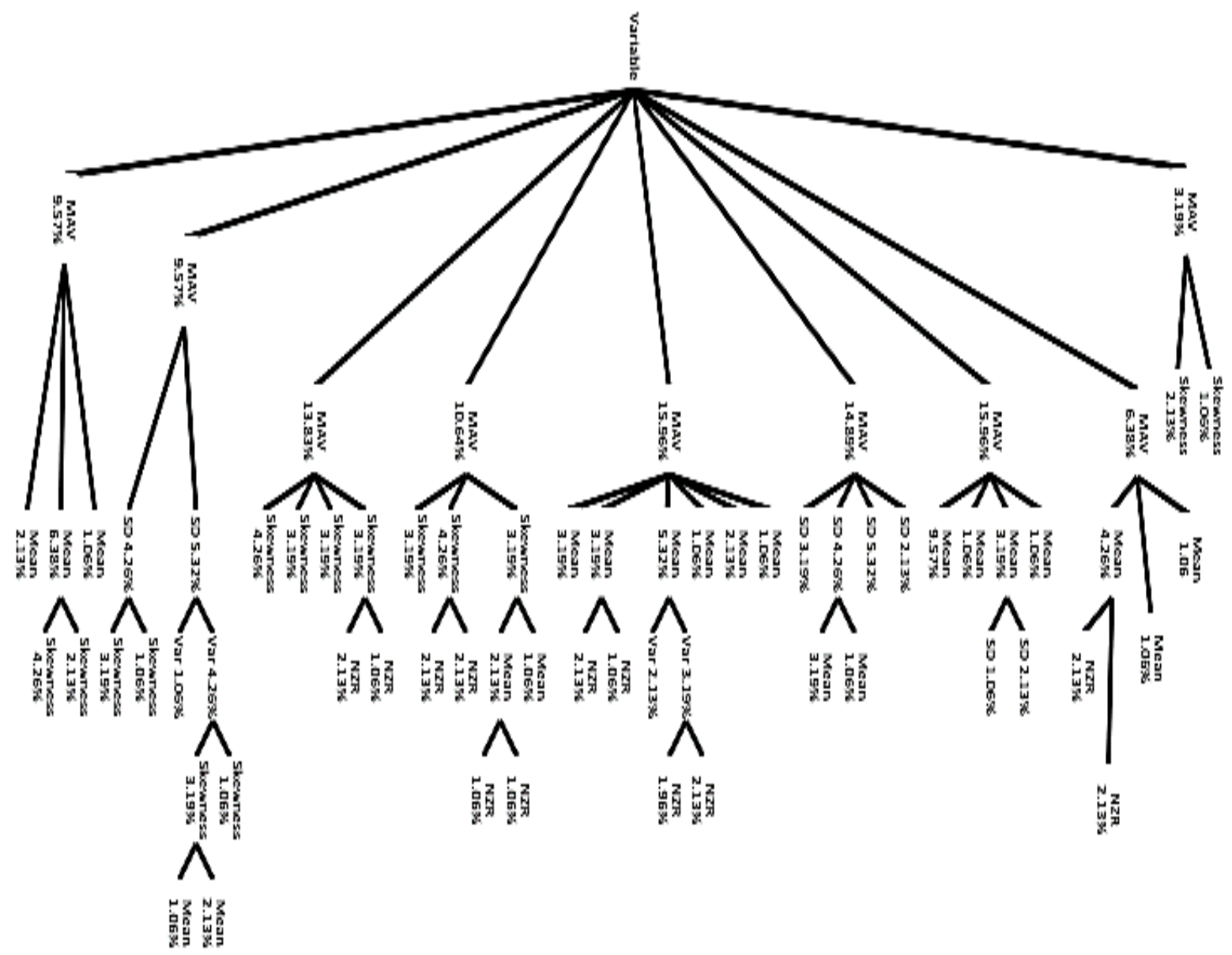

Figure 7. Tree diagram

Table 1. Confusion Matrix for the Training Sample

\begin{tabular}{llllll}
\hline From/To & Paper & Rock & Scissors & Total & Accuracy \% \\
\hline Paper & 22 & 2 & 3 & 27 & 81.48 \\
Rock & 0 & 25 & 2 & 27 & 92.59 \\
Scissors & 6 & 5 & 29 & 40 & 72.50 \\
Total & 28 & 32 & 34 & 94 & 80.85 \\
\hline
\end{tabular}

\section{CONCLUSION}

In this paper, we described a proof-of-concept methodology to recognize three hand gestures which is typically used as a sign language for rehabilitation. The data collected by sEMG. Time domain feature was used for human activity recognition. sEMG exhibit signal patters that are distinctive for each hand gesture. The data was extracted into several features which are mean, MAV, standard deviation, variance, skewness, NZR and RMS. The features were feed into classifier which are PCA and classification and regression tree. Both classifiers were compared. It was found that PCA obtained accuracy of $72.59 \%$ while, regression tree has accuracy of $80.85 \%$. There is still needed an improvement for the experiment. 


\section{REFERENCES}

[1] B. Milosevic et al, "Design Challenges for Wearable EMG Applications," Design, Automation \& Test in Europe Conference \& Exhibition (DATE), Lausanne, pp. 1432-1437. 2017.

[2] A. L. Rincon et al, "Design of a Video Game for Rehabilitation using Motion Capture, EMG Analysis and Virtual Reality," 2016 International Conference on Electronics, Communications and Computers (CONIELECOMP), Cholula, 2016, pp. 198-204.

[3] J. Wu et al, "A Wearable System for Recognizing American Sign Language in Real-time using IMU and Surface EMG Sensors," IEEE Journal of Biomedical adnd Health Informatics, vol 20(5), pp. 1281-1290, 2016.

[4] K. O. Thielbar et al., "Benefits of Using a Voice and EMG-Driven Actuated Glove to Support Occupational Therapy for Stroke Survivors," in IEEE Transactions on Neural Systems and Rehabilitation Engineering, vol. 25, no. 3, pp. 297-305, March 2017.

[5] J. Connolly et al, "IMU Sensor-based Electronic Goniometric Glove for Clinical Finger Movement Analysis," in IEEE Sensors Journal, vol. 18(3), pp. 1273-1281, 1 Feb.1, 2018.

[6] P. Hsiao et al, "Data Glove Embedded with 9-axis IMU and Force Sensing Sensors for Evaluation of Hand Function," 2015 37th Annual International Conference of the IEEE Engineering in Medicine and Biology Society (EMBC), Milan, 2015, pp. 4631-4634.

[7] H. Ahmed et al, "Improving the Accuracy of Human Body Orientation Estimation with Wearable IMU Sensors," in IEEE Transactions on Instrumentation and Measurement, vol. 66, no. 3, pp. 535-542, March 2017.

[8] V. Vidya et al, "IMU Sensor based Self Stabilizing Cup for Elderly and Parkinsonism," 2017 International Conference on Advances in Computing, Communications and Informatics (ICACCI), Udupi, 2017, pp. 2264-2269.

[9] J. Wu et al, "A Wearable System for Recognizing American Sign Language in Real-Time Using IMU and Surface EMG Sensors," IEEE Journal of Biomedical and Health Informatics, 20(5), 1281-1290, 2016.

[10] S. Negi et al, "Feature Extraction and Classification for EMG Signals using Linear Discriminant Analysis," 2016 2nd International Conference on Advances in Computing, Communication, \& Automation (ICACCA) (Fall), Bareilly, 2016, pp. 1-6.

[11] D. Zhang et al, "A Comparative Study on PCA and LDA based EMG Pattern Recognition for Anthropomorphic Robotic Hand," 2014 IEEE International Conference on Robotics and Automation (ICRA), Hong Kong, 2014, pp. 4850-4855.

[12] Ç. Çerçi and H. Temeltaş, "Feature extraction of EMG Signals, Classification with ANN and kNN Algorithms," 2018 26th Signal Processing and Communications Applications Conference (SIU), Izmir, 2018, pp. 1-4.

[13] S. Negi et al, "Feature Extraction and Classification for EMG Signals using Linear Discriminant Analysis," 2016 2nd International Conference on Advances in Computing, Communication, \& Automation (ICACCA) (Fall), Bareilly, 2016, pp. 1-6.

[14] C. Li, et al, "The Signal Processing and Identification of Upper Limb Motion Based on sEMG," Wireless Personal Communications, vol 103(1), pp. 887-896, 2018.

[15] O. Bai et al, "Characterization of Lower Limb Activity during Gait using Wearable, Multi-channel Surface EMG and IMU Sensors," 2017 International Electrical Engineering Congress (iEECON), Pattaya, 2017, pp. 1-4.

[16] Z. Liu et al., "A Novel Upper Limb Training System Based on UR5 using sEMG and IMU Sensors," 2016 IEEE International Conference on Robotics and Biomimetics (ROBIO), Qingdao, 2016, pp. 1069-1074.

[17] A. Bhattacharya et al, "Time Domain Multi-Feature Extraction and Classification of Human Hand Movements using Surface EMG," 2017 4th International Conference on Advanced Computing and Communication Systems (ICACCS), Coimbatore, 2017, pp. 1-5.

[18] T. Funabashi et al, "On-line Recognition of Finger Motions using Wrist EMG and Simple-PCA," 2015 10th Asian Control Conference (ASCC), Kota Kinabalu, 2015, pp. 1-5.

[19] W. Caesarendra et al, "EMG based Classification of Hand Gestures using PCA and ANFIS," 2017 International Conference on Robotics, Biomimetics, and Intelligent Computational Systems (Robionetics), Bali, 2017, pp. 18-23.

[20] W. Caesarendra et al, "A Classification Method of Hand EMG Signals based on Principal Component Analysis and Artificial Neural Network," 2016 International Conference on Instrumentation, Control and Automation (ICA), Bandung, 2016, pp. 22-27.

[21] S. A. Fandakh et al, "A Fast and Highly Accurate EMG Signal Classification Approach for Multifunction Prosthetic Fingers Control," $40^{\text {th }}$ International Conference Telecommunication and Signal Processing (TSP), pp. 395-398, 2017 IEEE.

[22] M. Haris, et al, "EMG Signal Based Finger Movement Recognition for Prosthetic Hand Control," 2015 Communication, Control and Intelligent Systems (CCIS), Mathura, 2015, pp. 194-198.

[23] L. Liu et al, "Development of an EMG-ACC-Based Upper Limb Rehabilitation Training System," in IEEE Transactions on Neural Systems and Rehabilitation Engineering, vol. 25, no. 3, pp. 244-253, March 2017.

[24] X. Liu et al, "The Use of a Finger-Worn Accelerometer for Monitoring of Hand use in Ambulatory Settings," IEEE Journal of Biomedical and Health Informatics, 1-1, 2018.

[25] Y. C. Du et al, "An IMU Compensated Skeletal Tracking System using Kinect for the Upper Limb," Microsystem Technologies, vol 24(10), pp. 4317-4327, 2018. 


\section{BIOGRAPHIES OF AUTHORS}
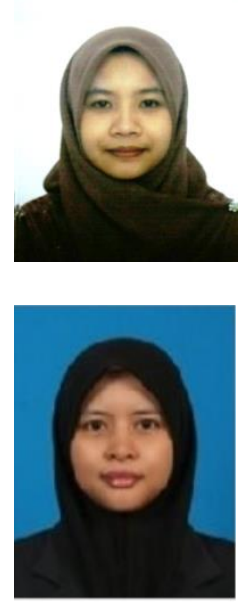

Najla Ilyana A.M received the B.S. degree in Electrical-Electronic Engineering from Universiti Teknologi Malaysia in 2016 and she is currently enrolled in the M.S. degree in Faculty of Electrical Engineering at Universiti Teknologi Malaysia. Her current research interests are in the area of medical electronic engineering including wireless rehabilitation using wearable device and monitoring engineering.

N. A Zakaria received his B.Eng degree in Electronic and System Engineering University of Takushoku, Tokyo, Japan in 2004. After graduation, she spent a year as an Industrial Engineering at Engineering Department in Pioneer Technology (M) Sdn Bhd. From 2006 she has served as a tutor in Universiti Teknologi Malaysia (UTM), before pursuing her M.Eng. (Electronics \& Communications) in UTM. During her study, her research area related to biomedical engineering field, which is focused on electroencephalography signal analysis for epilepsy patient prediction. In April 2011, she started her ph.D study in Faculty of Biomedical Engineering in Chiba University, Chiba, Japan, before continuing her works in Information Science Department in Nara institute of Science and Technology, Nara, Japan. She received her ph.D degree in September, 2019 focusing on research fall risk analysis among elderly, rehabilitation engineering. Since 2014, she is serving at the Faculty of Electrical Engineering, which currently known as School of Electrical Engineering, Faculty of Engineering, UTM. Within the Faculty of Electrical Engineering at the UTM, she teaches a few courses related to her research and elementary to the electrical and electronics curriculum. 